\title{
Plant Disease Detection using Image Processing
}

\author{
${ }^{1}$ Mr.V Suresh, ${ }^{2} \mathrm{D}$ Gopinath, ${ }^{3} \mathrm{M}$ Hemavarthini, ${ }^{4} \mathrm{~K}$ Jayanthan, ${ }^{5}$ Mohana Krishnan \\ ${ }^{1}$ Assistant Professor, CSE Department, Dr.NGP Institute Of Technology \\ 2,3,4,5 UG Scholar CSE Department, Dr.NGP Institute Of Technology
}

\begin{abstract}
Identification of the plant diseases is the key to prevent the losses in the yield and quantity of the agricultural product. The studies of the plant diseases mean the studies of visually observable patterns seen on the plant. Health monitoring and disease detection on plant is very critical for sustainable agriculture. It is very difficult to monitor the plant diseases manually. It requires tremendous amount of work, expertise in the plant diseases, and also require the excessive processing time. Hence, image processing is used for the detection of plant diseases by capturing the images of the leaves and comparing it with the data sets. The data set consist of different plant in the image format. Apart from detection users are directed to an e-commerce website where different pesticides with its rate and usage directions are displayed. This website can be efficiently used for comparing the MRP's of different pesticides and purchase the required one for the detected disease. This paper aims to support and help the green house farmers in an efficient way.
\end{abstract}

Keywords: Plant disease detection, Tensor flow, Green house, Convolution neural network, Data model, image to byte code

\section{INTRODUCTION}

India is a cultivated country and about $70 \%$ of the Population depends on agriculture. Farmers have large range of diversity for selecting various suitable crops and finding the suitable pesticides for plant. Hence, damage to the crops would lead to huge loss in productivity and would ultimately affect the economy.

Leaves being the most sensitive part of plants show disease symptoms at the earliest. The crops need to be monitored against diseases from the very first stage of their life-cycle to the time they are ready to be harvested. Initially, the method used to monitor the plants from diseases was the traditional naked eye observation that is a time-consuming technique which requires experts to manually monitor the crop fields. In the recent years, a number of techniques have been applied to develop automatic and semi-automatic plant disease detection systems and automatic detection of the diseases by just seeing the symptoms on the plant leaves makes it easier as well as cheaper. These systems have so far resulted to be fast, inexpensive and more accurate than the traditional method of manual observation by farmers

In most of the cases disease symptoms are seen on the leaves, stem and fruit. The plant leaf for the detection of disease is considered which shows the disease symptoms.

There are many cases where farmers do not have a fully compact knowledge about the crops and the disease that can get affected to the crops. This paper can be effectively used by farmers thereby increasing the yield rather than visiting the expert and getting their advice.

The main objective is not only to detect the disease using image processing technologies. It also directs the user directly to an e-commerce website where the user can purchase the medicine for the detected disease by comparing the rates and use appropriately according to the directions given.

Greenhouse also called a glasshouse, or, if with sufficient heating, a hoth house, is a structure with walls and roof made chiefly of transparent material, such as glass, in which plants requiring regulated climatic conditions are grown. As greenhouse farming is gaining more importance now a day's, this paper helps the greenhouse farmers in an effective way. Various techniques can be used to review the plant disease detection and discuss in terms of various parameters. The paper is organized into the following sections. First section gives a brief introduction to the importance of plant disease detection. Second section discusses the existing work carried out recently in this area and also reviews the techniques used. Section three includes methodologies used in our paper. Lastly, fourth section concludes this paper along with future directions.

\section{LITERATURE SURVEY}

Alternia leaf spot, Brown spot, Mosaic, Grey spot, and Rust are five common types of apple disease that severly affect apple yield. However, the existing research lacks an accurate and fast detector of apple disease for ensuring the healthy development of the apple industry. Object detection algorithms such as SSD, DSSD and R-SSD can be regarded as consisting of two parts: The first part is the pre-network model, which is used as a basic features extractor. The other is an auxillary structure that utilizes multi-scale feature map for a detection[1].

A K-means segmentation is used for partitioning the leaf image into four clusters using the squared Euclidean distances. The method applied for feature extraction is Colour Co-occurrence method for both colour and texture features[4]. Finally, classification is completed using neural network detection algorithm based on back propagation methodology. The overall system disease detection and classification accuracy was found to be around $93 \%$.

A number of crop types namely, fruit crops, vegetable crops, cereal crops and commercial crops to detect fungal diseases on plant leaves. Different methods have been adopted for each type of crop[5].For fruit crops, $\mathrm{k}$-means clustering is the segmentation method used, 
texture features have been focused on and classified using ANN and nearest neighbour algorithms achieving an overall average accuracy of $90.723 \%$. For vegetable crops, chan-vase method used for segmentation, local binary patterns for texture feature extraction and SVM and knearest neighbour algorithm for classification achieving an overall average accuracy of $87.825 \%$.

The commercial crops have been segmented using grab-cut algorithm. Wavelet based feature extraction has been adopted using Mahalnobis distance and PNN as classifiers with an overall average accuracy of $84.825 \%$

The cereal crops have been segmented using kmeans clustering and canny edge detector. Colour, shape, texture, colour texture and random transform features have been extracted. SVM and nearest neighbour classifiers used getting an overall average accuracy of $83.72 \%$.A chilli plant leaf image and processed to determine the health status of the chilli plant. Their technique is ensuring that the Chemicals should apply to the diseased chilli plant only. They used the MATLAB for the feature extraction and image recognition. In this paper pre-processing is done using the.

Fourier filtering, edge detection and morphological operations. Computer vision extends the image processing paradigm for object classification. Here digital camera is used for the image Capturing and LABVIEW software tool to build the GUI[7].

The FPGA and DSP based system is developed and used for monitoring and control of plant diseases. The FPGA is used to get the field plant image or video data for monitoring and diagnosis.

The DSP TMS320DM642 is used to process and encode the video or image data. The nRF24L01 single chip $2.4 \mathrm{GHz}$ radio transmitter is used for data transfer. It has two data compress and transmission method to meet user's different need and uses multi-channel wireless communication to lower the whole system cost.

\section{METHODS OF DISEASE DETECTION}

The process of plant disease detection system basically involves four phases as shown in Fig 3.1. The first phase involves acquisition of images either through digital camera and mobile phone or from web. The second phase segments the image into various numbers of clusters for which different techniques can be applied. Next phase contains feature extraction methods and the last phase is about the classification of diseases.

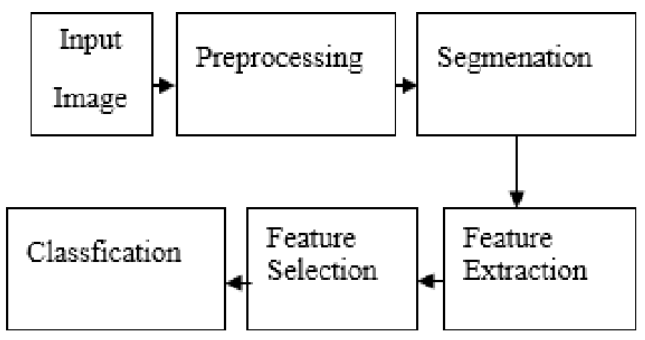

\section{Image Acquisition}

In this phase, images of plant leaves are gathered using digital media like camera, mobile phones etc. with desired resolution and size. The images can also be taken from web.

The formation of database of images is completely dependent on the application system developer. The image database is responsible for better efficiency of the classifier in the last phase of the detection system.

\section{Image Segmentation}

This phase aims at simplifying the representation of an image such that it becomes more meaningful and easier to

Analyze. As the premise of feature extraction, this phase is also the fundamental approach of image processing.

There are various methods using which images can be segmented such as k-means clustering, Otsu's algorithm and thresholding etc. The k-means clustering classifies objects or pixels based on a set of features into $\mathrm{K}$ number of classes. The classification is done by minimizing the sum of squares of distances between the objects and their corresponding clusters.

\section{Feature Extraction}

Hence, in this step the features from this area of interest need to be extracted. These features are needed to determine the meaning of a sample image.

Features can be based on colour, shape, and texture. Recently, most of the researchers are intending to use texture features for detection of plant diseases. There are various methods of feature extraction that can be employed for developing the system such as gray-level co-occurrence matrix (GLCM), color cooccurrence method, spatial greylevel dependence matrix, and histogram based feature extraction. The GLCM method is a statistical method for texture classification.

\section{Classification}

The classification phase implies to determine if the input image is healthy or diseased. If the image is found to be diseased, some existing works have further classified it into a number of diseases. For classification, a software routine is required to be written in MATLAB, also referred to as classifier.

A number of classifiers have been used in the past few years by researchers such as k-nearest neighbour (KNN), support vector machines (SVM), artificial neural network(ANN), back propagation neural network (BPNN), Naïve Bayes and Decision tree classifiers.

The most commonly used classifier is found to be SVM. Every classifier has its advantages and disadvantages, SVM is simple to use and robust technique. 


\section{OVERVIEW OF PLANT DISEASE}

Plant diseases are generally caused by infectious agents such as fungi, bacteria, and viruses. Signs of plant disease are observable evidence of infection and symptoms are the visible effects of these kinds of disease. Fungal infections cause signs like visible spores, mildew, or mold and the basic symptoms are like leaf spot and yellowing.

Fungal diseases are plant infections caused by fungi. Fungi can be single or multicellular, but either way infect plants by stealing nutrients and breaking down tissue. Fungal diseases are the most common infection in plants. There are some characteristic symptoms, or observable effects of the disease, in plants.

Fungi infections can be recognized by symptoms like spots on plant leaves, yellowing of leaves, and birdseye spots on berries. With some fungal diseases, the organism itself can actually be viewed on the leaves appear as a growth and as a mold

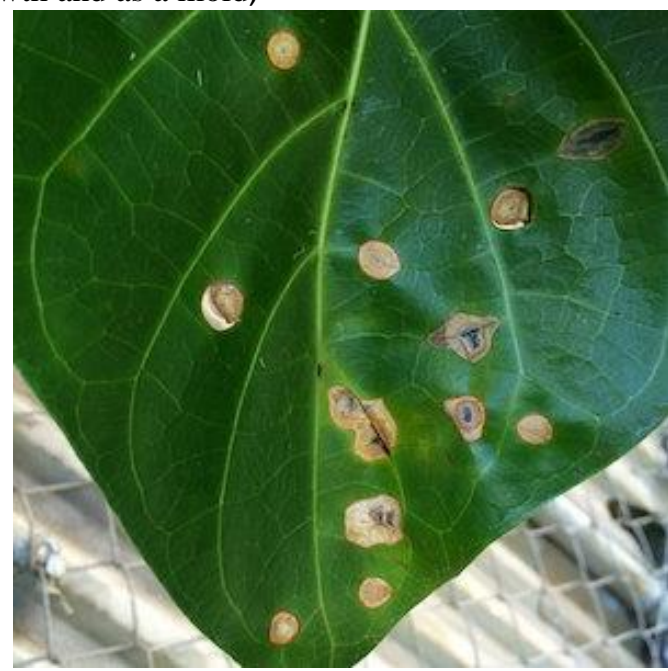

Fig 4.1 Leaf affected by fungal infection

These may a malformations on stems or the underside of leaves. These direct observations of the disease-causing organism are called signs of infection Bacteria are single-celled, prokaryotic organisms. Bacteria are everywhere and many can be beneficial, but some can cause disease both in humans and plants.

The signs of bacteria are often harder to detect than fungi, since bacteria are microscopic. Upon cutting an infected stem, a milky white substance may appear, called bacterial ooze. This is one sign of a bacterial infection. Other signs include water-soaked lesions, which are wet spots on leaves that ooze bacteria.

Eventually, as the disease progresses, the lesions enlarge and form reddish-brown spots on the leaves. A common symptom of bacterial infection is leaf spots or fruit spots. Unlike fungal spots, these are often contained by veins on the leaf.

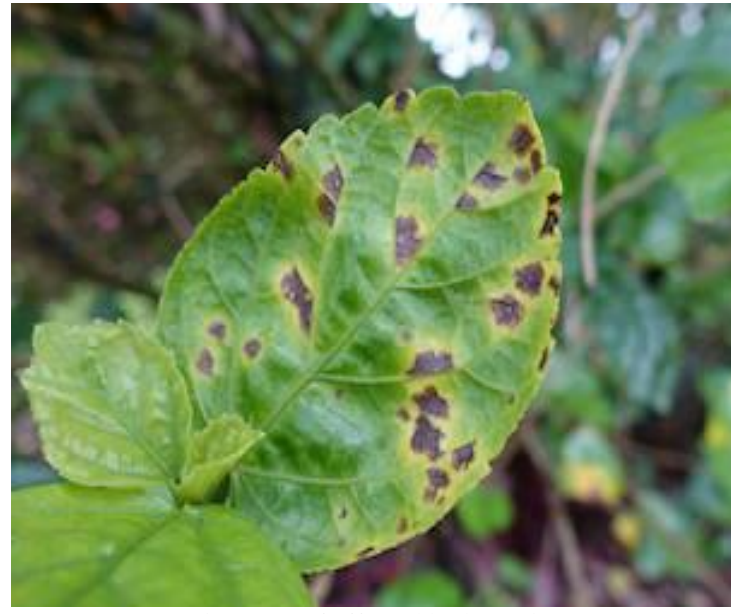

Fig 4.2 Leaf affected by bacteria

Viruses are infectious particles that are too small to be detected by a light microscope. They invade host cells and hijack host machinery to force the host to make millions of copies of the virus.

Viral diseases don't show any signs in plants since viruses themselves cannot be seen even with a light microscope. However, there are symptoms that the trained eye can observe. A mosaic leaf pattern, yellowed, or crinkled leaves are all

characteristic of viral infection. This classic pattern of discoloration is where many plant viruses get their name, such as the tobacco mosaic virus. Also, decreased plant growth is also commonly seen in viral infections.

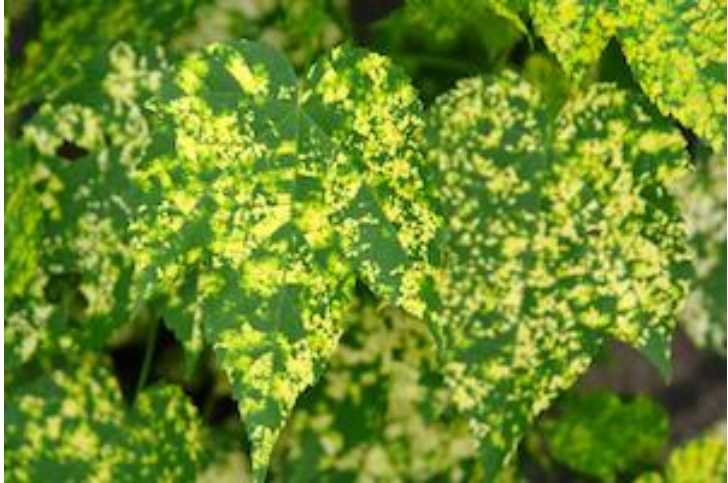

Fig 4.3 Leaf affected by virus

So, these are our observation on how to classify the various plant disease and how to be cautious about that.

\section{PROPOSED SYSTEM}

Proposed system have an end-to-end Android application with TFLite. Proposed system opted to develop an Android application that detects plant diseases. It has the algorithms and models to recognize species and diseases in the crop leaves by using Convolutional Neural Network. Proposed system use Colab to edit source code.

A dataset of 54,305 images of diseased and healthy plant leaves collected under controlled conditions Plant Village dataset. The images cover 14 species of crops, including: apple, blueberry, cherry, grape, orange, peach, 
pepper, potato, raspberry, soy, squash, strawberry and tomato. It contains images of 17 basic diseases, 4 bacterial diseases, 2 diseases caused by mold (oomycete), 2 viral diseases and 1 disease caused by a mite. 12 crop species also have healthy leaf images that are not visibly affected by disease. Our dataset contains solutions for several plant textures such as,

\section{Apple scab}

2. Apple black rot

3. Apple cedar apple rust

4. Cherry including sour powdery mildew

5. Cherry including sour healthy

6. Corn maize cercospora leaf spot gray leaf spot

7. Corn maize common rust

8. Corn maize northern leaf blight

9. Corn maize healthy

10. Grape black rot

11. Grape esca black measles

12. Grape leaf blight isariopsis leaf spot

13. Grape healthy

14. Orange haunglongbing citrus greening

15. Peach bacterial spot

16. Peach healthy

17. Pepper bell bacterial spot

18. Pepper bell healthy

19. Potato early blight

20. Potato late blight

21. Squash powdery mildew

22. Strawberry leaf scorch

23. Tomato bacterial spot

24. Tomato early blight

25. Tomato late blight

26. Tomato leaf mold

27. Tomato septoria leaf spot

28. Tomato spider mites two spotted spider mite

29. Tomato target spot

30. Tomato yellow leaf curl virus

31.Tomato mosaic virus

Data generators that will read pictures in our source folders, convert them to 'float32' tensors, and feed them (with their labels) to our network is set up.

As data that goes into neural networks should usually be normalized in some way to make it more amenable to processing by the network. In our case, we will pre-process our images by normalizing the pixel values to be in the ' $[0$, 1] range (originally all values are in the ' $[0,255]$ range). We will need to make sure the input data is resized to $224 \times 224$ pixels or $299 \times 299$ pixels as required by the networks. You have the choice to implement image augmentation or not.

Apart from just detecting the plant disease using the above methods our system directs the user to an ecommerce website. This website displays all the pesticides that are available for the detected disease with its MRP rate. Along with this the directions to use it is also available in the website. Thus by comparing the rate and features of the pesticides the user can purchase it

\section{RESULTS AND DISCUSSION}

There two different conditions for training and testing. One is under the lab conditions, which means that the model is tested with the images from the same dataset from which it is used for both training and testing. The other condition is that field condition; this means that our model has tested with the images taken from the real world conditions (land). Since the lighting conditions and background properties of the images are totally different when we take samples from the real field, there is a chance that our model to produce a very low accuracy, when comparing to the accuracy values acquired during the lab conditions. So to overcome this impact, we had an idea of having a mixed variety of images during the training phase (heterogeneity).
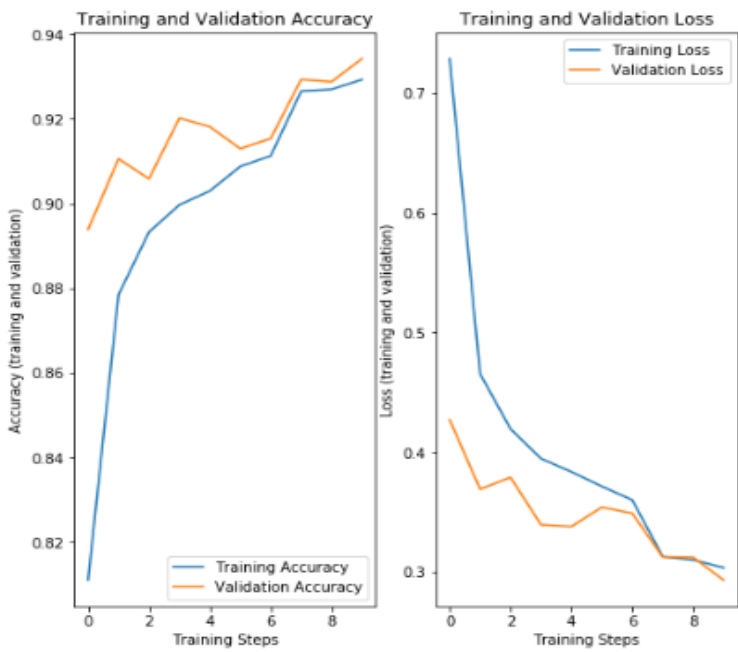

Fig.6.1 Performance

The accuracy of Real-time detection of apple leaf disease using deep learning approach based on improved convolution neural networks is less when compared to the proposed system because it detects multiple diseases in a single system.

\begin{tabular}{|c|c|c|}
\hline $\begin{array}{c}\text { Input } \\
\text { (apple) }\end{array}$ & $\begin{array}{c}\text { Faster R- } \\
\text { CNN }\end{array}$ & Proposed system \\
\hline Scab & 58.82 & 70.82 \\
\hline Black rot & 68.12 & 82.68 \\
\hline Cedar apple rust & 90.34 & 94.96 \\
\hline
\end{tabular}

\section{CONCLUSION}

Thus an application built for the identification of disease affected plants and healthy plants is done and this proposed work is focuses on the accuracy values during the real field conditions, and this work is implemented by having several plant disease images.

Overall this work is implemented from scratch and produces a decent accuracy. The future work is to increase the number of images present in the predefined database and to modify the architecture in accordance with the dataset for achieving better accuracy. 


\section{REFERENCES}

[1] Peng Jiang, Yuehan Chen, Bin Liu, Dongjian He, Chunquan Liang ,' Real-Time Detection of Apple Leaf Diseases Using Deep Learning Approach Based on Improved Convolutional Neural Networks', ( Volume: 7 ), pp. 06 May 2019

[2] Zhou, R., Kaneko, S., Tanaka, F., Kayamori, M., Shimizu, M., 'Disease detection of Cercospora Leaf Spot in sugar beet by robust template matching', Computers and Electronics in Agriculture, Volume 108, pp.58-70, 2014

[3] Barbedo, J.G.A., Godoy, C.V., 'Automatic Classification of Soybean Diseases Based on Digital Images of Leaf Symptoms', SBI AGRO,2015

[4] Barbedo, J.G.A., 'A review on the main challenges in automatic plant disease identification based on visible range images', 2016 ,Biosystems Engineering, Volume 144, pp. 52-60.

[5] Bashish, D.A., Braik, M., Ahmad, S.B., 'A Framework for Detection and Classification of Plant Leaf and Stem Diseases', International Conference on Signal and Image Processing, pp. $113-118,2010$
[6] Punajari, J.D., Yakkundimath, R., Byadgi, A.S., 'Image Processing Based Detection of Fungal Diseases In Plants', International Conference on Information and Communication Technologies, Volume 46, pp. 1802-1808, 2015

[7] Zulkifli Bin Husin, Abdul Hallis Bin Abdul Aziz, Ali Yeon Bin Md Shakaff Rohani Binti S Mohamed Farook, "Feasibility Study on Plant Chili Disease Detection Using Image Processing Techniques", 2012 Third International Conference on Intelligent Systems Modelling and Simulation.

[8] Chunxia Zhang, Xiuqing Wang, Xudong Li, "Design of Monitoring and Control Plant Disease System Based on DSP\&FPGA", 2010 Second International Conference on Networks Security, Wireless Communications and TrustedComputing.

[9] Omrani, E., Khoshnevisan, B., Shamshirband, S., Saboohi, H., Anuar, N.B., Nasir, M.H.N., 'Potential of radial basis functionbased support vector regression for apple disease detection', Journal of Measurement, pp. 233-252, 2014

[10] Gharge, S., Singh, P., 'Image Processing for Soybean Disease Classification and Severity Estimation', Emerging Research in Computing, Information, Communication and Applications, pp. 493-500, 2016 